\title{
Biobehavioural Physiotherapy through Telerehabilitation during the SARS-CoV-2 Pandemic in a Patient with Post-polio Syndrome and Low Back Pain: A Case Report
}

\author{
Alberto García-SALGAdo, MSc ${ }^{1}$ and Mónica Grande-Alonso, $\mathrm{PhD}^{1,2}$ \\ ${ }^{1)}$ Instituto de Rehabilitación Funcional La Salle, Spain \\ ${ }^{2)}$ Physical Therapy Department, Centro Superior de Estudios Universitarios La Salle, Universidad Autónoma de Madrid, Spain
}

\begin{abstract}
Post-polio syndrome refers to the physical and psychological sequelae caused by poliovirus infection. For this reason, according to which the emotional and sensorimotor sphere is affected, we consider a biobehavioural approach based on education and therapeutic exercise to be necessary. The aim of this case report is to evaluate the effect of a biobehavioural approach in a patient with post-polio syndrome and low back pain. We describe a 57-year-old man with post-polio syndrome and low back pain following a fall at the end of February 2020. The pain, disability and lack of functionality caused by both processes led him to contact a physiotherapy service. A therapeutic planning was carried out for 3 months, where a biobehavioural approach based on therapeutic exercise and education, with an assessment and three face-to-face sessions which were complemented by online follow-up and finalised due to the Sars-Cov-2 pandemic in a telerehabilitation approach. It was organised in two phases; the initial phase lasted 2 weeks with the aim of reducing the symptoms of the lumbar region, and the advanced phase in which the aim was to improve his physical condition. During the three-month intervention, four assessments were conducted (Pre, at 4 weeks, at 8 weeks and at 12 weeks). At follow-up, improvements in functional and psychological variables were obtained. This case suggests that a biobehavioural approach through telerehabilitation was a useful option in this reported case and could be an option of treatment to improve psychological, physical and functional variables in this patient.
\end{abstract}

Key words: Post-polio syndrome, Biobehavioural physiotherapy, Therapeutic alliance, Therapeutic exercise, Biobehavioural strategies

(Phys Ther Res 24: 295-303, 2021)

P which caused an epidemic that began with a high infection rate in the 1940s and lasted until the 1970s, mainly affecting children younger than 5 years and spreading through the oral and faecal routes. A high percentage of those infected are typically asymptomatic; however, some patients start with central nervous system symptoms that cause

Received: February 10, 2021

Accepted: June 22, 2021

Advance Publication by J-STAGE: September 3, 2021

Correspondence to: Mónica Grande-Alonso, Physical Therapy Department, Centro Superior de Estudios Universitarios La Salle, Universidad Autónoma de Madrid, Spain, Madrid Madrid C/ La Salle $n^{\circ}$ 10, 28023, Spain

\# e-mail: monica.grande@irflasalle.es

doi: 10.1298/ptr.E10100 acute flaccid paralysis ${ }^{1}$. Survivors of this epidemic can experience a late onset of new symptoms and a worsening of sequelae after a mean of approximately 15 years, which leads to a series of symptoms that determine the so-called post-polio syndrome (PPS), which include muscular weakness and reduced muscular resistance, accompanied by fatigue, atrophy and musculoskeletal pain ${ }^{1,2)}$. The pathogenesis is not well defined, but the most currently supported hypothesis describes these sequelae as a metabolic depletion of motor units, caused by an increase in motor neuron size in response to distal axon reinnervation, coupled with an inflammatory process with high concentrations of proinflammatory cytokines in the cerebrospinal fluid ${ }^{2,3)}$.

The physiotherapeutic approach to PPS patients is essential, with the aim of delaying functional deterioration. Functionality may have been affected in the population due 
to the Sars-Cov-2 pandemic, lack of physical activity due to confinement in the months of March to May and the increase in home work which further encourages this sedentary lifestyle, increasing musculoskeletal deterioration and decreasing aerobic capacity, and leading to increased insulin resistance and inflammatory states ${ }^{4}$. So telerehabilitation has become important. A recent systematic review concludes that active approaches using telerehabilitation have shown comparable clinical outcomes to conventional faceto-face rehabilitation approaches ${ }^{5}$. However, for patients with PPS, the literature is contradictory regarding the choice of exercise modality, as strength or aerobic exercises have shown little or no improvement in functional tests ${ }^{6}$.

Currently, a biobehavioural approach aims to change the experience of pain based on biological, behavioural and cognitive systems ${ }^{7)}$. This is defined as an approach in which the patient must be an active processor and agent in order to generate adaptive learning. It takes into account how cognitive, individual and environmental factors influence physiological processes and the experience of pain ${ }^{8,9)}$. Focusing treatment on education and therapeutic exercise (TE) through telerehabilitation could be more useful in patients with PPS. It allows both the medical interview and the development of objectives and treatment to be carried out telematically, being a cheap and effective resource. Teleassistance allows remote contact and the possibility of communicating in a specific time and being able to resolve questions directly, addressing all the factors that may intervene in the treatment of PPS. That influence this pathology, but it has been observed that there is a lack of evidence regarding the application of this approach in patients with PPS. The main objective of the biobehavioural approach is to provide the patient with active coping strategies and thus to empower him/her and to develop an increased internal locus of control ${ }^{10)}$. Research studies on patients with chronic musculoskeletal pain have shown that techniques such as education or TE can be included in the biobehavioural approach as they increase self-efficacy and improve somatosensory, motor and psychological variables ${ }^{11-13)}$.

This is the first case study that evaluates a biobehavioural physiotherapy treatment by means of telerehabilitation in the described clinical picture. Therefore, the purpose of this study was to evaluate and treat a patient with back pain and PPS through a biobehavioural approach based on the patient centred biopsychosocial model.

\section{Case Presentation}

The study was conducted in accordance with the Declaration of Helsinki and the informed consent form was signed by the patient prior to the start of the procedure.

We report the case of a 57-year-old male patient (weight, $70 \mathrm{~kg}$; height, $170 \mathrm{~cm}$ ) who presented a PPS and low-back pain after a fall a week previously. His pathologi- cal history included hypertension and post-polio sequelae. He had contracted polio when he was 5 months of age and had an operation on each foot at the age of 8 years, due to an excessive deformity of the plantar arch of the foot, normalising the cavus foot position and lengthening the plantar tendons with a subsequent cast for 3 months.

The patient is currently taking a medication based on 2.5-12.5 mg of ramipril-hydrochlorothiazide for arterial hypertension and $100 \mathrm{mg}$ of aspirin due to an ocular microinfarct with a rise in blood pressure.

The patient reported an initial episode of pain that occurred 2 days prior to the initial evaluation, in which there was an increase in walking-related pain when supporting his weight on the right leg. Also reported a second pain in the transition from sitting to standing. The symptoms of the initial episode of low back pain were described as deep pain of a mechanical nature, with the sensation of puncture located in the right lumbar area, with an intensity of $50 \mathrm{~mm}$ on the visual analogue scale (VAS), which increased with physical effort to $70 \mathrm{~mm}$ on the VAS. The second pain was described as superficial, diffuse pain, located in the left lumbar region, referred to as a constant pressure that radiated towards the lateral face and went down the leg, with an intensity of $40 \mathrm{~mm}$ on the VAS. Figure 1.1 shows the body chart of the initial assessment with the representation of the symptoms, adding a third intermittent episode of pain in the left foot with articular characteristics and another intermittent episode of pain in both popliteal holes with myotendinous characteristics.

\section{Physical examination}

After the medical interview, a physical examination was performed. The patient was asked to perform active trunk flexion movements, which caused discomfort on the left side; during the extension, the first episode of pain described above was evoked at the end of the range. There was no pain during the passive physiological movements, but the accessory physiological movements on the right side, located on the lumbar facets L3 and L4, reproduced his pain described as deep and prick. During palpation, the left paravertebral musculature presented local hyperalgesia with the referred symptoms described in figure 1.1.

The patient subsequently performed functional tests aimed at static balance, given that he mentioned having a falling frequency of twice a month.

The patient first performed the Functional Reach Test, which has been proven to be valid and reliable for evaluating static stability ${ }^{14}$. The physiotherapist measured the postural stability in the forward direction, obtained through the movement achieved by the patient (in $\mathrm{cm}$ ) by shifting the centre of gravity to the limits of the support base, while the feet remained stationary. The evaluation employed a measuring device consisting of a measuring tape that was placed at the height of the patient's acromion. The patient placed 

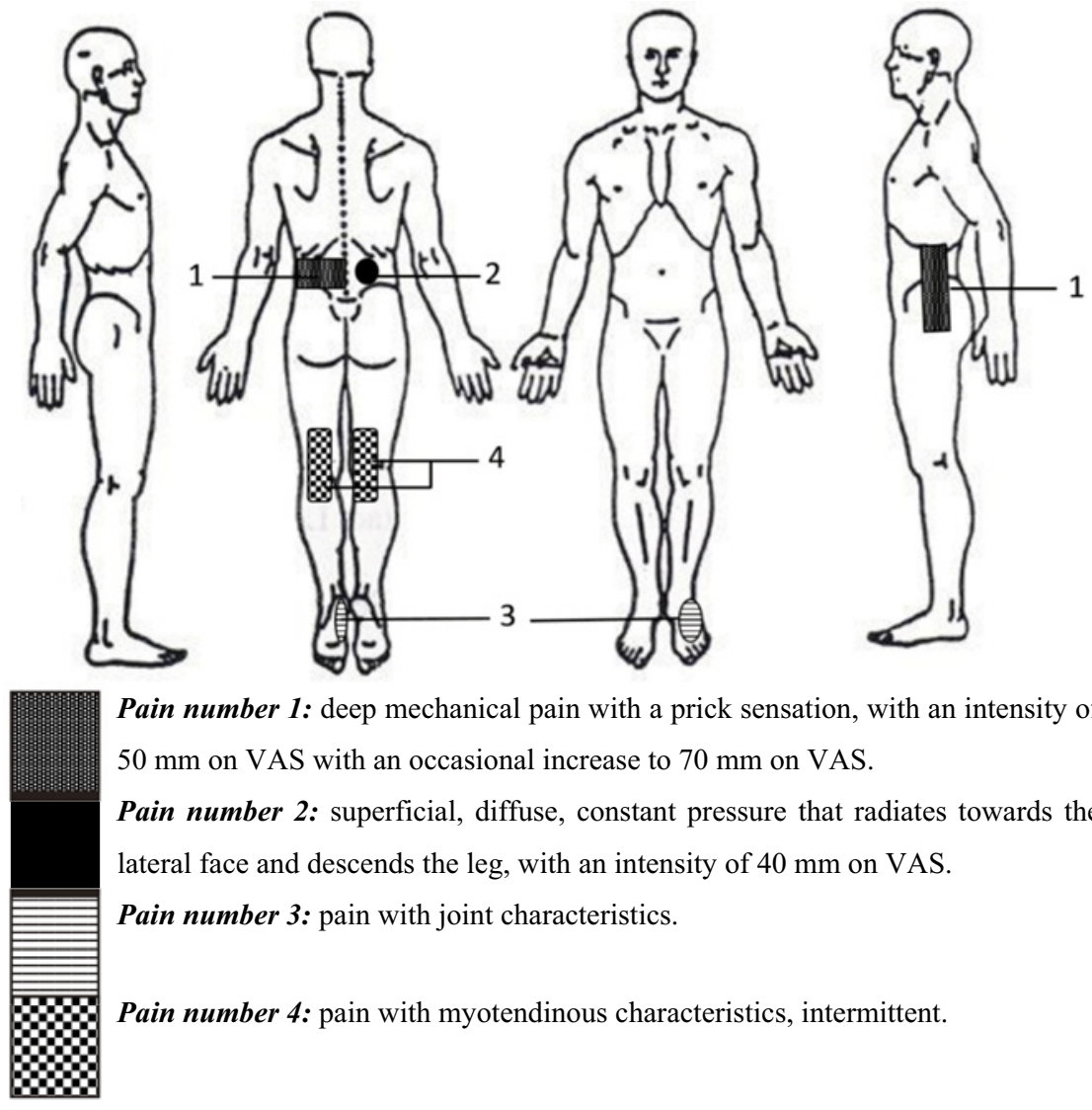

Pain number 1: deep mechanical pain with a prick sensation, with an intensity of $50 \mathrm{~mm}$ on VAS with an occasional increase to $70 \mathrm{~mm}$ on VAS.

Pain number 2: superficial, diffuse, constant pressure that radiates towards the lateral face and descends the leg, with an intensity of $40 \mathrm{~mm}$ on VAS.

Pain number 3: pain with joint characteristics.

Pain number 4: pain with myotendinous characteristics, intermittent.

Fig. 1.1. Pre-intervention body chart with symptom distribution.

both arms at 90 degrees of flexion, with the elbows and hands at full extension. He then had to reach as far as possible while maintaining the posture for 2-3 seconds without lifting his feet off the ground ${ }^{14,15)}$.

The patient's capacity for effort was evaluated by means of the 6-minute walk test (6MWT), a general purpose functional test for cardiac and respiratory diseases, which has been proven to be valid and reliable. Provides information as a follow-up measure of the functional change in an activity such as walking, employed in combination with the modified scale of perceived effort ${ }^{16)}$. In this case, the test was modified so that the number of steps taken during this time and the effort involved were counted on the Borg scale, employing a wrist pedometer ${ }^{17}$.

Next, the evaluation of fear of movement was performed using the 11-item Spanish version of the Tampa Scale of Kinesiophobia (TSK-11), whose reliability and validity have been demonstrated ${ }^{18)}$. The TSK-11 consists of 2 subscales: 1 related to fear of physical activity and the other related to fear of harm. The final score can range from 11 to 44 points, with higher scores indicating greater perceived kinesiophobia ${ }^{18)}$.

Finally, functionality was assessed using the Lower Limb Functional Index (LLFI) questionnaire, a selfregistration tool used as an outcome measure that assesses bodily functions, activities, participation factors, and the environment both at the beginning of an intervention and the result of said treatment. The questionnaire has 25 items in Likert format, with an internal consistency of $0.91^{19)}$.

Four measurements were taken: the first was a preintervention test (T0), and the rest were undertaken every 4 weeks (T1, T2, T3). The last 3 measurements were performed telematically in the same manner as the initial assessment by the patient, given that the environmental conditions did not change; these measurements were supervised by a physiotherapist via videoconference. The measurements from the LLFI and TSK-11 self-recording questionnaires were only taken at $\mathrm{T} 0$ and $\mathrm{T} 3$.

\section{Therapeutic intervention}

The patient underwent 4 measurement days (MD), 3 physical therapy sessions on-site during the first two weeks (PT), 4 therapeutic patient education sessions (TPE) and 32 TE sessions lasting 60 minutes over 3 months were conducted, divided in two routines: strength training (ST) and cardiorespiratoryresistance training (CRT), carried out with a frequency of two days per week each. During the first two weeks, 6 sessions based on exercise for low back pain (ELBP) were carried out. Appendix 1 specifies the treatment followed during the process, while Table 1 shows the established planning. A treatment model based on the biobehavioural paradigm focusing on therapeutic education 


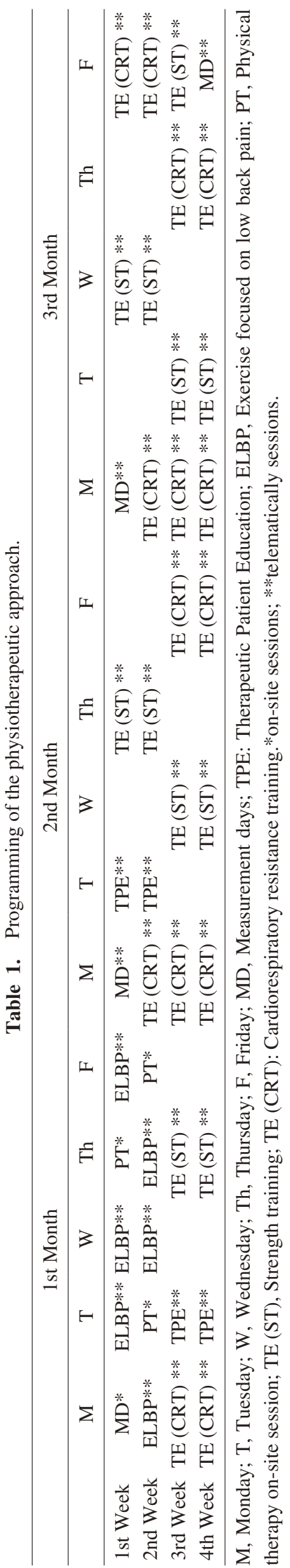

and TE was used. This paradigm is defined as one in which the patient must be an active processor and agent in order to generate adaptive neuroplastic changes, thus providing the patient with active coping strategies ${ }^{8,9}$.

On the first day of the intervention, the initial assessment and test treatment were carried out. During the second week, the planned approach was continued and two face-toface sessions were required for pain management and organisation of the TE programmes. The 3 on-site sessions (PT) focused on managing low back pain with manual therapy, as well as prescribing TE based on physical reconditioning, combined with therapeutic education focusing on the benefits of exercise based on a Strengths, Weaknesses, Opportunities and Threats (SWOT) analysis (Fig. 2). The approach through this analysis is to present to the patient what Strengths (physical, motivation, desire to change, etc.) and Weaknesses (pain, functionality, hopelessness, control, strength deficit, etc.) he had and to elaborate a way to address them with tools, transforming them into an opportunity or therapeutic strategy. No longer seeing these weaknesses as a fragile pillar but as a motivation for change. And to expose threats from an objective point of view (how likely is an event to happen just because you believe it? educate in maladaptive beliefs, etc.).

The remaining sessions were conducted telematically via a video call system due to the SARS-Cov-2 pandemic, in which the methodology to be followed was presented by means of a table of contents in slides. The online follow-up time was divided into 60 minutes in which the topic of therapeutic education in pain and exercise neurophysiology was presented, as well as different coping and motivational strategies. A SWOT model was used within a biobehavioural paradigm in order to address all patient needs.

\section{Therapeutic patient education}

Therapeutic education is a tool that aims to change maladaptive beliefs and erroneous thoughts that interfere with the patient's pathology and pain perception, which lead to an increase in the presence of psychological variables such as fear of movement ${ }^{20,21}$. Research studies on patients with chronic musculoskeletal pain have shown that the application of the technique leads to significant improvements in variables such as disability, fear of movement, catastrophizing, anxiety and physical fitness ${ }^{22-24}$. Four sessions were conducted, one session per week between the third and sixth week of treatment. The intervention covered topics related to the benefits of physical activity, the influence of psychosocial factors and maladaptive beliefs.

\section{Therapeutic exercise}

TE were defined as a physical exercise plan designed and prescribed to facilitate recovery from illness or any impairment of movement and activities of daily living ${ }^{25,26)}$. Research has shown that a low level of physical activity can 


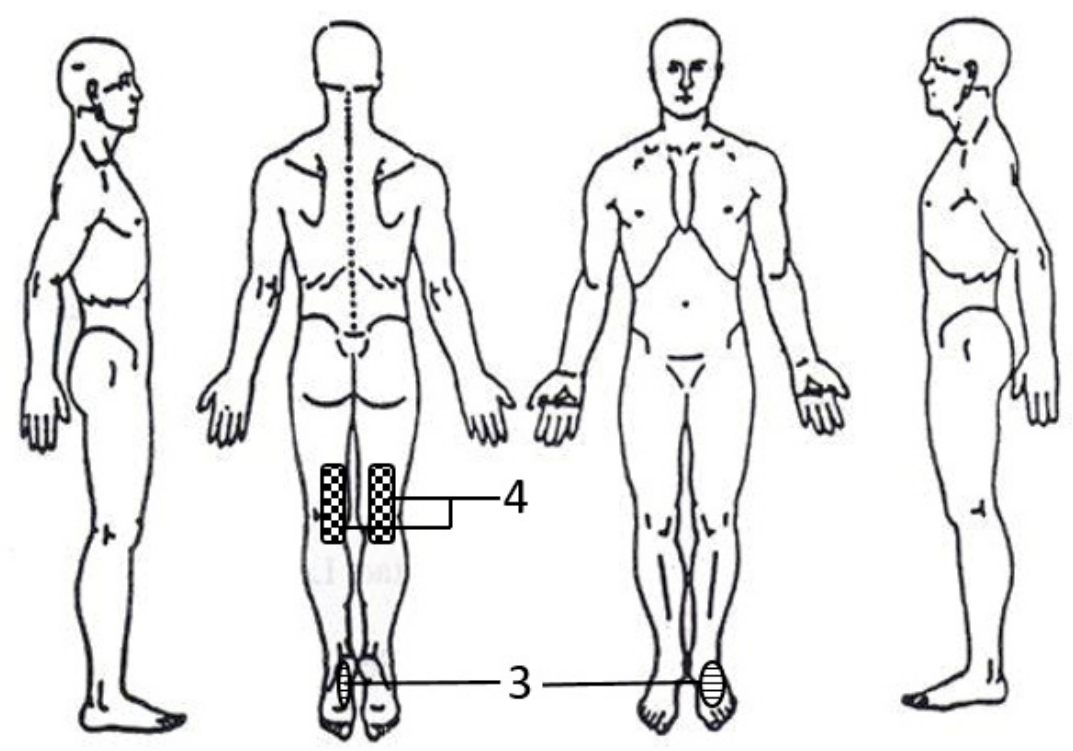

Pain number 3: pain with joint characteristics.

Pain number 4: pain with myotendinous characteristics, intermittent.

Fig. 1.2. Post-intervention body chart with symptom distribution.

Table 2. Baseline and follow-up measurements from T0 to T3

\begin{tabular}{lrrrr}
\hline \multicolumn{1}{c}{ Measure } & T0 & T1 & T2 & T3 \\
\hline TSK-11 (points) & 33 & - & - & 14 \\
FRT (cm) & 24 & 23 & 25 & 25 \\
6MWT (steps) & 645 & 651 & 663 & 668 \\
6MWT (meters) & 490 & 496 & 505 & 508 \\
Modified Borg Scale from 0 to 10 points & 6 & 6 & 7 & 6 \\
LLFI (percentage of functionality) & $56 \%$ & - & - & $64 \%$ \\
Pain number 1 (VAS) & 5 & 3 & 1 & 0 \\
Pain number 2 (VAS) & 4 & 1 & 0 & 0 \\
\hline
\end{tabular}

T0, baseline measurement; T1, measurement at the $4^{\text {th }}$ week; T2, measurement at the $8^{\text {th }}$ week; T3, measurement at the $12^{\text {th }}$ week; TSK-11, Tampa Scale of Kinesiophobia; FRT, Functional Reach Test; 6MWT, 6-minute walk test; LLFI, Low Limb Functional Index; VAS, Visual analog scale.

lead to reduced neuromuscular efficiency and decreased strength, resulting in a number of negative consequences on functionality and postural control ${ }^{27-29)}$. On the basis of the above, we consider it necessary to implement a TE programme based on strength and cardiorespiratory endurance exercise for 12 weeks at a frequency of 2-3 times a week.

\section{Results}

The results are shown in Table 2. The patient showed a improvement between the T0 and T3 measurements in terms of kinesiophobia level (from 33 to 14 points), with a reported minimum detectable change of 5.46 points considered relevant ${ }^{30)}$, as well as improvement in the LLFI from
$56 \%$ to $64 \%$ of functionality ${ }^{31)}$. In terms of pain intensity, there was also a improvement, exceeding the minimum detectable change for the first reported episode of pain from $50 \mathrm{~mm}$ to $0 \mathrm{~mm}$ and in the second reported episode of pain from $40 \mathrm{~mm}$ to $0 \mathrm{~mm}^{32}$. Figure 1.2 demonstrates the improvement after three months of intervention in terms of pain expansion using the body chart.

Static stability showed a slight improvement. The available evidence indicates that, for the 50-59-year age range, the distance in the Functional Reach Test should be $27.6 \pm 6.6 \mathrm{~cm}^{33}$. The patient showed an improvement in the variable of distance walked during the 6MWT, but he did not exceed the minimum detectable change in this population for the test. In relation to the $6 \mathrm{MWT}$, the patient pre- 


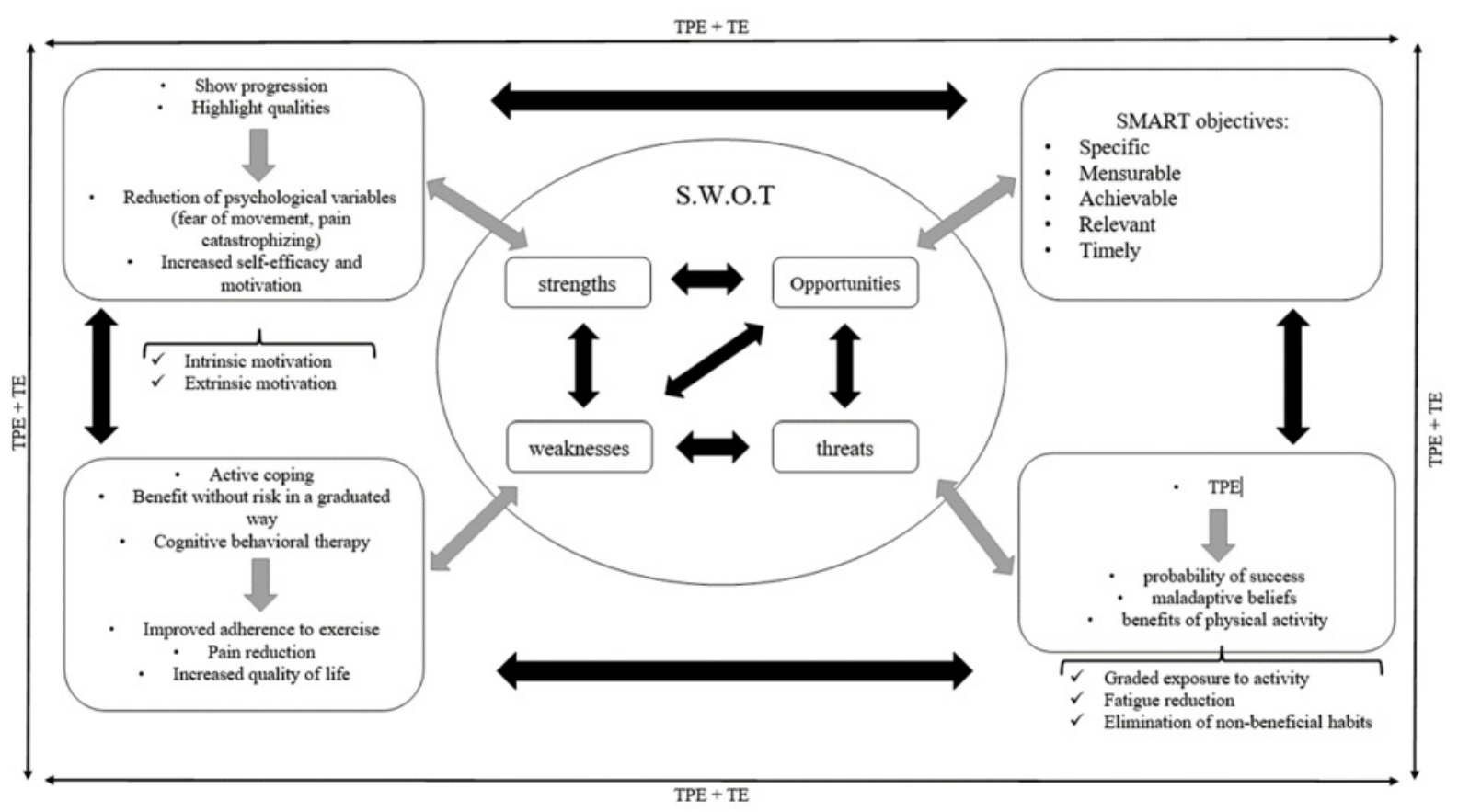

Fig. 2. S.W.O.T analysis for therapeutic approach

TE: Therapeutic exercise; TPE: Therapeutic Patient Education.

sented the same perceived fatigue for the T0 and T3 measurements.

\section{Discussion}

The reported case shows an evaluation and treatment option from a biobehavioural paradigm of a patient with PPS and low back pain, with the aim of improving functionality. Currently, there is a lack of scientific evidence on biobehavioural physiotherapy treatment in PPS, even more so on the approach of this type of patient with physiotherapy through telerehabilitation. There is a growing use of telematic intervention and online assessment, an excellent low-cost tool ${ }^{34)}$, that is limited by the lack of interpersonal proximity. It is therefore crucial to employ strategies that increase the patient's self-efficacy and adherence to $\mathrm{TE}$, given this is a primary pillar of treatment. We recommend using indicators during the session that provide a better therapeutic alliance, such as reinforcing positive actions, planning real objectives and the process towards achieving them and providing instruction that supports selfmanagement ${ }^{35)}$. Moore et al. in their study stated important barriers that interfere with the success of exercise therapy, such as the existence of pain itself, low self-efficacy and lack of motivation, as well as various long-term facilitators for patient improvement that help patients to adhere to exercise. Describing "exercise awareness" as a pillar that benefits and prevents episodes of pain, modifying such exercises if necessary according to the patient's perception and thus improving their self-efficacy by having greater capabilities and allowing them to do so even when in pain ${ }^{36)}$.
And finally the "therapeutic alliance" with its three characteristics of warmth, collaboration and support, coupled with skills, emotion management and communication, being the therapeutic alliance an activity to be practised ${ }^{36,37)}$. Showing empathy and authenticity during the session and monitoring the therapist-patient relationship by facilitating 2-way feedback will foster such a therapeutic alliance by creating a positive emotional bond that will lead to greater adherence to fulfilling the planned goals ${ }^{38-40)}$.

One of the most powerful strategies in physical therapy with greater impact on increased self-efficacy is performing TE. Based on the current literature, however, the evidence from certain modalities, such as aerobics and strength training, is contradictory due to the heterogeneity and lack of studies published on this population ${ }^{2,3)}$. Regardless, the neurophysiological and functional benefits of TE are well known in general terms, even in other complex painful or pathological conditions, such as sarcopenia and chronic fatigue syndrome, in which there is also a lack of homogeneity in research studies ${ }^{41-44)}$.

Based on these results, we set up a TE programme according to the effort perceived by the patient as an active strategy from a cognitive-behavioural perspective, accompanied by manual therapy techniques and therapeutic education, using an approach based on a SWOT analysis, which highlights the strengths of the programme.

In this way, the ability to resolve an activity is demonstrated by highlighting the quality of the action, addressing the weaknesses to be overcome and using an approach that shows the way forward by setting specific objectives.

This methodology has been employed as a clinical 
guideline when making decisions in other health areas ${ }^{45)}$.

Although cognitive-behavioural therapy has not been studied in this population with respect to pain, the effect of such therapy on variables such as fatigue has been studied, but no beneficial effect has been reported ${ }^{46)}$. The biobehavioural approach has been shown to have beneficial effects in various populations with chronic pain by reducing pain intensity and improving quality of life ${ }^{47)}$, addressing aspects of the bio-psycho-social sphere by intervening in maladaptive beliefs, expectations, self-efficacy and motivation ${ }^{48-51)}$.

The results of this intervention were positive with respect to reducing the intensity of low back pain improvement in kinesiophobia, balance, number of steps and metres walked in 6 minutes, as well as data obtained from the LLFI. This intervention comprised a physical therapy approach based on a biobehavioural paradigm centred on the prescription of exercise and on obtaining tools that promote self-management and adherence to exercise.

There are several limitations in this study to consider, the first of which was the lack of monitoring during the exercise, given that monitoring helps provide greater motivation and easier correction of the proposed activities. Second, the recovery time required by the patient due to the major fatigue induced by performing the exercise sessions made it necessary to reduce the volume of training and increase the resting time. The third limitation was the lack of heart rate monitoring during the activities and the lack of anthropometric measurements. Another important limitation is the lack of assessment of adherence and the patient's subjective perception of improvement after treatment, which could provide more clinical information and, as the study by Moore et al., found, may have a direct effect on motivation $^{36)}$. The last limitations were the lack of knowledge of the long-term effectiveness and that of each intervention, given that different strategies were presented during the process, and the lack of reproducibility due to the variability of the measurements taken at T0 (face-to-face) and the rest of the measurements that were guided and collected remotely.

\section{Conclusion}

In conclusion, focusing treatment on a biobehavioural paradigm centred on TE and education throught telerehabilitation can be a treatment option, as observed in this patient with PPS and musculoskeletal pain, as it can lead to improvements in the medium term on functional and psychological variables.

Conflict of Interest: The authors declare that they have no competing interests.

Acknowledgments: The authors thank the Centro Superior de Estudios Universitarios (CSEU) La Salle for mak- ing this study possible and for their help with translation corrections of the manuscript.

\section{References}

1) Jones KM, Balalla $S$, et al:: A systematic review of the worldwide prevalence of survivors of poliomyelitis reported in 31 studies. BMJ Open. 2017; 7: e015470.

2) Koopman FS, Beelen A, et al.: Treatment for postpolio syndrome. Cochrane database Syst Rev. 2015; CD007818.

3) Lo JK and Robinson LR: Post-polio syndrome and the late effects of poliomyelitis: Part 2. treatment, management, and prognosis. Muscle Nerve. 2018; 58: 760-769.

4) Bowden Davies KA, Pickles S, et al.: Reduced physical activity in young and older adults: metabolic and musculoskeletal implications. Ther Adv Endocrinol Metab. 2019; 10.

5) Suso-Martí L, La Touche R, et al:: Effectiveness of Telerehabilitation in Physical Therapist Practice: An Umbrella and Mapping Review with Meta-Meta-Analysis. Phys Ther. February 2021.

6) Stolwijk-Swüste JM, Beelen A, et al.: The course of functional status and muscle strength in patients with late-onset sequelae of poliomyelitis: a systematic review. Arch Phys Med Rehabil. 2005; 86: 1693-1701.

7) Henschke N, Ostelo RW, et al.: Behavioural treatment for chronic low-back pain. Cochrane Database Syst Rev. 2010; 2010.

8) O'Sullivan PB, Caneiro JP, et al.: Cognitive functional therapy: An integrated behavioral approach for the targeted management of disabling low back pain. Phys Ther. 2018; 98: 408-423.

9) Flor H and Turk DC: Chronic Pain: An Integrated Biobehavioral Approach. IASP (Press). 2011.

10) Turk DC: Cognitive-Behavioral Approach to the Treatment of Chronic Pain Patients. Reg Anesth Pain Med. 2003; 28: 573579.

11) Marcos-Martín F, González-Ferrero L, et al. : Multimodal physiotherapy treatment based on a biobehavioral approach for patients with chronic cervico-craniofacial pain: a prospective case series. Physiother Theory Pract. 2018; 34: 671-681.

12) López-de-Uralde-Villanueva I, Beltran-Alacreu H, et al.: Pain management using a multimodal physiotherapy program including a biobehavioral approach for chronic nonspecific neck pain: a randomized controlled trial. Physiother Theory Pract. 2020; 36: 45-62.

13) Grande-Alonso M, Suso-Martí L, et al.: Physiotherapy Based on a Biobehavioral Approach with or Without Orthopedic Manual Physical Therapy in the Treatment of Nonspecific Chronic Low Back Pain: A Randomized Controlled Trial. Pain Med. 2019; 20: 2571-2587.

14) Duncan PW, Weiner DK, et al:: Functional reach: a new clinical measure of balance. J Gerontol. 1990; 45: M192-M197.

15) Newton RA: Validity of the multi-directional reach test: a practical measure for limits of stability in older adults. J Gerontol A Biol Sci Med Sci. 2001; 56: M248-M252.

16) Du H, Newton PJ, et al:: A review of the six-minute walk test: its implication as a self-administered assessment tool. Eur J Car- 
diovasc Nurs. 2009; 8: 2-8.

17) Barker KL, Dawes H, et al.: Perceived and measured levels of exertion of patients with chronic back pain exercising in a hydrotherapy pool. Arch Phys Med Rehabil. 2003; 84: 1319-1323.

18) Gómez-Pérez L, López-Martínez AE, et al.: Psychometric Properties of the Spanish Version of the Tampa Scale for Kinesiophobia (TSK). J Pain. 2011; 12: 425-435.

19) Cuesta-Vargas AI, Gabel CP, et al.: Cross cultural adaptation and validation of a Spanish version of the Lower Limb Functional Index. Health Qual Life Outcomes. 2014; 12: 75.

20) Nijs J, Paul van Wilgen C, et al.: How to explain central sensitization to patients with "unexplained" chronic musculoskeletal pain: Practice guidelines. Man Ther. 2011; 16: 413-418.

21) Fletcher C, Bradnam L, et al.: The relationship between knowledge of pain neurophysiology and fear avoidance in people with chronic pain: A point in time, observational study. Physiother Theory Pract. 2016; 32: 271-276.

22) Louw A, Diener I, et al.: The effect of neuroscience education on pain, disability, anxiety, and stress in chronic musculoskeletal pain. Arch Phys Med Rehabil. 2011; 92: 2041-2056.

23) Wood L and Hendrick PA: A systematic review and metaanalysis of pain neuroscience education for chronic low back pain: Short-and long-term outcomes of pain and disability. Eur J Pain (United Kingdom). 2019; 23: 234-249.

24) Moseley GL: Evidence for a direct relationship between cognitive and physical change during an education intervention in people with chronic low back pain. Eur J Pain. 2004; 8: 39-45.

25) World Health Organization: (2015). Physical activity. Fact sheet 385.

26) Kottke FJ, Stillwell GK, et al:: Krusen's Handbook of Physical Medicine and Rehabilitation, Saunders, 1982.

27) Hicks GE, Gaines JM, et al.: Associations of back and leg pain with health status and functional capacity of older adults: Findings from the retirement community back pain study. Arthritis Care Res. 2008; 59: 1306-1313.

28) Brech GC, Andrusaitis SF, et al.: Correlation of disability and pain with postural balance among women with chronic low back pain. Clinics. 2012; 67: 959-962.

29) Benavent-Caballer V, Sendín-Magdalena A, et al.: Physical factors underlying the Timed "Up and Go" test in older adults. Geriatr Nurs (Minneap). 2016; 37: 122-127.

30) Hapidou EG, O'Brien MA, et al.: Fear and Avoidance of Movement in People with Chronic Pain: Psychometric Properties of the 11-Item Tampa Scale for Kinesiophobia (TSK-11). Physiother Can. 2012; 64: 235-241.

31) Gabel CP, Melloh M, et al:: Lower limb functional index: development and clinimetric properties. Phys Ther. 2012; 92: 98-110.

32) Ostelo RWJG and de Vet HCW: Clinically important outcomes in low back pain. Best Pract Res Clin Rheumatol. 2005; 19: 593607.

33) Tantisuwat A, Chamonchant D, et al.: Multi-directional Reach Test: An Investigation of the Limits of Stability of People Aged between 20-79 Years. J Phys Ther Sci. 2014; 26: 877-880.

34) Fatoye F, Gebrye T, et al.: The Clinical and Cost-Effectiveness of Telerehabilitation for People With Nonspecific Chronic Low Back Pain: Randomized Controlled Trial. JMIR mHealth uHealth. 2020; 8: e15375.

35) Williams SL and French DP: What are the most effective intervention techniques for changing physical activity self-efficacy and physical activity behaviour--and are they the same? Health Educ Res. 2011; 26: 308-322.

36) Moore AJ, Holden MA, et al.: Therapeutic alliance facilitates adherence to physiotherapy-led exercise and physical activity for older adults with knee pain: a longitudinal qualitative study. J Physiother. 2020; 66: 45-53.

37) Babatunde F, MacDermid J, et al.: Characteristics of therapeutic alliance in musculoskeletal physiotherapy and occupational therapy practice: A scoping review of the literature. BMC Health Serv Res. 2017; 17.

38) Kinney M, Seider J, et al.: The impact of therapeutic alliance in physical therapy for chronic musculoskeletal pain: A systematic review of the literature. Physiother Theory Pract. 2020; 36: 886898.

39) Pihlaja S, Stenberg J-H, et al.: Therapeutic alliance in guided internet therapy programs for depression and anxiety disorders - A systematic review. Internet Interv. 2018; 11: 1-10.

40) Nienhuis JB, Owen J, et al.: Therapeutic alliance, empathy, and genuineness in individual adult psychotherapy: A meta-analytic review. Psychother Res. 2018; 28: 593-605.

41) Elsawy B and Higgins KE: Physical activity guidelines for older adults. Am Fam Physician. 2010; 81: 55-59.

42) Papa E V, Dong X, et al.: Resistance training for activity limitations in older adults with skeletal muscle function deficits: a systematic review. Clin Interv Aging. 2017; 12: 955-961.

43) Brightwell CR, Markofski MM, et al.: Moderate - intensity aerobic exercise improves skeletal muscle quality in older adults. Transl Sport Med. 2019; 2: 109-119.

44) Larun L, Brurberg KG, et al.: Exercise therapy for chronic fatigue syndrome. Cochrane database Syst Rev. 2017; 4 : CD003200.

45) Von Kodolitsch Y, Bernhardt AM, et al.: Analysis of Strengths, Weaknesses, Opportunities, and Threats as a Tool for Translating Evidence into Individualized Medical Strategies (I-SWOT). Aorta (Stamford, Conn). 2015; 3: 98-107.

46) Walklet E, Muse K, et al: : Do Psychosocial Interventions Improve Quality of Life and Wellbeing in Adults with Neuromuscular Disorders? A Systematic Review and Narrative Synthesis. J Neuromuscul Dis. 2016; 3: 347-362.

47) Beltran-Alacreu H, Lopez-de-Uralde-Villanueva I, et al.: Multimodal Physiotherapy Based on a Biobehavioral Approach as a Treatment for Chronic Tension-Type Headache: A Case Report. Anesthesiol pain Med. 2015; 5: e32697.

48) Touche R La, Grande-Alonso M, et al.: How does self-efficacy influence pain perception, postural stability and range of motion in individuals with chronic low back pain? Pain Physician. 2019; 22: E1-E13.

49) Jung MJ and Jeong Y: Motivation and Self-Management Behavior of the Individuals With Chronic Low Back Pain. Orthop Nurs. 2016; 35: 330-337. 
50) Bushnell MC, Ceko M, et al.: Cognitive and emotional control of pain and its disruption in chronic pain. Nat Rev Neurosci. 2013; 14: 502-511.

51) Navratilova E, Morimura K, et al.: Positive emotions and brain reward circuits in chronic pain. J Comp Neurol. 2016; 524:
1646-1652.

Supplementary material (Appendix):

1. Appendix 1. Therapeutic intervention 